\title{
Diagramas y esquemas en y sobre la genética mendeliana*
}

Diagrams and Schemes in and about Mendelian Genetics

Mario Casanueva ${ }^{\dagger \neq}$

\begin{abstract}
Resumen
A pesar de su importancia creciente los diagramas, esquemas y bocetos aún son un territorio relativamente poco explorado en la filosofía de la biología. Se presenta un diagrama, en formato grafo-modelo, que recupera la estructura conceptual de la genética mendeliana. Este diagrama se usa como herramienta de análisis epistemológico de otro diagrama que puede ser considerado paradigmático en la genética clásica: el cuadro de Punnett.
\end{abstract}

Palabras clave: Punnett - grafo - modelo - diagrama - representación - visualización - estructura lógica de las teorías

\begin{abstract}
Despite their growing importance, diagrams, schemes and sketches are still a relatively unexplored territory in the philosophy of biology. We present a diagram, in graph-model format, that recovers the conceptual structure of Mendelian genetics. This diagram is used as a tool for epistemological analysis of another diagram that can be considered paradigmatic in Classical genetics: The Punnett Square.
\end{abstract}

Keywords: Punnett - graph - model - diagram - representation - visualization - logical structure of theories

* Recibido: 30 de Marzo de 2016. Aceptado con revisiones: 5 de Diciembre de 2016.

† Departamento de Humanidades, Phibio, REMO, Universidad Autónoma Metropolitana-Cuajimalpa. Para contactar al autor, por favor, escribir a: mario.casanueva@gmail.com.

* Agradezco a Diego Méndez, Octavio Valadez, Ximena González y Rubén Madrigal sus comentarios a una versión previa. Este trabajo participa de los proyectos CB 182084, del CONACYT, México, y PICT-2012-2662 y PICT-2014-1741 de la ANPCyT, Argentina. Metatheoria 8(1)(2017): 83-93. ISSN 1853-2322.

(C) Editorial de la Universidad Nacional de Tres de Febrero. Publicado en la República Argentina. 
We ought to talk less and draw more. I personally should like to renounce speech altogether and, like organic nature, communicate everything I have to say in sketches.

Johann Wolfgang von Goethe ${ }^{1}$

\section{Sobre la importancia de los diagramas y su ponderación en biología y filosofía de la biología}

Animaciones, bocetos esquemas y diagramas, son útiles herramientas para la creación, manejo, adecuación, comunicación, transferencia y entendimiento de sistemas conceptuales y prácticos (Tversky et al. 2009, Tversky 2011). Actúan como instrumentos de representación y procesamiento de datos o relaciones de inferencia y constituyen herramientas cognitivas que asisten al razonamiento, a la solución de problemas y a la obtención de explicaciones de diversa índole (Wimsatt 2012, Tylén et al. 2014, Burnston et al. 2014, Abrahamsen \& Bechtel 2015, Burnston 2016). Permiten externalizar, fijar, vincular y recrear ideas y arquitecturas conceptuales (Tversky \& Suwa 2009), facilitan la transmisión de información condensada (densa) y son eficientes potenciadores heurísticos, e inductores de vías insospechadas para la relación de conceptos, contextos, perspectivas y niveles de profundidad de análisis (Larkin \& Simon 1987, Darden \& Craver 2002, Kozma 2003, Perini 2005, 2013, Ainsworth, Prain \& Tytler 2011, Jones \& Wolkenhauer 2012, Wimsatt 2012, Sheredos et al. 2013, Jones 2014).

El potencial heurístico de visualizaciones y diagramas en los procesos de generación y adecuación de modelos es una herramienta concomitante al desarrollo y trasmisión del conocimiento, pues las expresiones visuales, son anteriores al lenguaje y al texto, poseen analogías con ellos, y se encuentran vinculadas a las interconexiones espacio-abstracción-acción (Lakoff \& Johnson 1980, 1999, Tversky et al. 2000, Tversky \& Suwa 2009, Tversky 2011). Diagramas, bocetos e imágenes sesgan y canalizan nuestro pensamiento (Daston \& Gallison 1992, Galison 2006, Giere 2010, Vorms 2013) y son parte de nuestros recursos cognitivos que, junto a las teorías, los modelos y las metáforas, participan de prácticas cognitivas extendidas y soportan procesos de razonamiento construcción y aprehensión de conocimientos (Cheon 2014, Osbeck \& Nersessian 2014, Chandrasekharan \& Nersessian 2015). Adicionalmente, el despliegue de imágenes mentales implica el cumplimiento de exigencias de visualización (Mast \& Ellis 2015, Binder et al. 2014, Moreau 2012, Sollfrank et al. 2015), vinculadas con estados sensorio-motrices particulares (Aglioti Bufalari \& Candidi 2014, Ferri et al. 2012), lo que les confiere el carácter de experiencia multimodal y facilita su recuerdo y evocación.

Por otro lado, la biología moderna es impensable sin el uso de los diagramas, esquemas o imágenes. Basta hojear cualquier revista especializada en el campo para constatarlo. Piénsese en la importancia constitutiva que en sus respectivos contextos han tenido las imágenes de, por ejemplo, los árboles de pedigrí, en la genética clásica; los árboles de la vida, y dendogramas en la biología evolucionista; los mapas de destino celular, en embriología; los mapas cromosómicos, en la teoría cromosómica de la herencia; la representación diagramática de las vías metabólicas, en la bioquímica; las redes tróficas y los ciclos biogeoquímicos en ecología; la hélice alfa y la capa beta de las proteínas o la doble hélice del ADN, en la biología molecular; los diagramas florales, en botánica; etc. La relevancia que, en sus respectivos campos, posee esta mínima muestra de la diversidad de diagramas e imágenes usados en la biología, pone de manifiesto que es erróneo considerarlos como recursos secundarios o adicionales, finalmente prescindibles. Las numerosas inferencias que pueden "deducirse o extraerse" de cada una de ellas, inducen a pensarlas como modelos teóricos en sí mismos. ${ }^{2}$

\footnotetext{
${ }^{1}$ Citado en Rudwick (1976, p. 149), tomado a su vez de Auden y Mayer (1962, p. xv).

2 Los diagramas también han sido destacados como privilegiados objetos de estudio del cambio cultural Wimsatt (2012), llamó la atención sobre una serie de propiedades que les confieren ventajas a este respecto, sobre teorías o ideas: son claramente localizables y tienen
} 
No obstante, a diferencia del cambio ocurrido en la historiografía de la ciencia, donde el análisis de las imágenes esquemas y diagramas ha cobrado gran relevancia, ${ }^{3}$ en la filosofía de la ciencia, en general, o de la biología en particular, aún son relativamente pocos los territorios o escuelas que prestan atención al uso de imágenes y diagramas. Una clara excepción la constituye el llamado nuevo mecanicismo en biología, donde los diagramas se consideran importantes recursos del razonamiento útiles a la representación y la intervención (véase, entre otros, Bechtel \& Richardson 1993, Glennan 2002, Machamer, Darden \& Craver 2000, Bechtel \& Abrahamsen 2005, Perini 2005, 2013, Sheredos et al. 2013, Craver \& Kaiser 2013, Gebharter \& Kaiser 2013). Otras notorias excepciones provienen de la atención que algunos historiadores y filósofos de la biología han brindado a casos particulares, como los diagramas de Weismann (Griesemer \& Wimsatt 1989) o los cuadros de Punnett (Wimsatt 2012, Edwards 2012, 2016, Vorms 2013, 2014).

\section{Grafo modelo (semi-desglosado) de la estructura de la genética mendeliana ${ }^{4}$}

Dentro de la corriente semanticista en filosofía de la ciencia, las teorías empíricas se conciben como una clase o familia de modelos, mas no existe una manera única de caracterizarlos y se reconoce que ninguna posee un estatus privilegiado (Suppe 1977, van Fraassen 1970, Balzer, Moulines \& Sneed 1987, Giere 1988, 2004, 2010, Díez \& Moulines 2000, Casanueva 2005, Méndez \& Casanueva 2006, Zenker \& Gärdenfors 2015).

A continuación, se presenta la estructura de la genética mendeliana de manera, similar a como se hace en la teoría matemática de modelos. Prescindiendo del formalismo, se le considera como un ensamble arquitectónico de entidades (conjuntos) y funciones (morfismos). Las entidades se representan como íconos y las funciones como flechas. Las últimas pueden ser vistas como reglas de inferencia que permiten asignar o asociar entidades del destino a entidades del origen. La propuesta destaca (mediante varios tipos de flechas) diferentes papeles epistémicos que desempeñan las distintas partes del modelo en la obtención de respuestas a la pregunta que es objeto de la teoría. En este caso: ¿por qué la apariencia de los hijos puede ser semejante o diferente, de la de sus padres?, o, en otras palabras, ¿cómo se transmiten las características a lo largo de las generaciones?

fronteras bien delimitadas; son de fácil conteo y poseen características de fácil evaluación y comparación, por lo que permiten el empleo de métodos taxonómicos convencionales; su ascendencia es fácil de determinar; son relativamente independientes del contexto en el que se presentan; son altamente portables, lo que les confiere una alta capacidad de reproducción y migración hacia otros textos; su éxito en la comunicación de ideas es de fácil evaluación, pues los diagramas exitosos son multicopiados en diferentes textos; sirven como casos de razonamiento paradigmáticos; sus caracteres cruciales están sometidos a una intensa selección por lo que rápidamente estabilizan ciertos rasgos exitosos y poseen un alto grado de transmisión horizontal.

3 Considérese, por ejemplo, que "The Emergence of a Visual Language for Geological Science 1760-1840" de M. J. Rudwick (1976), es, a la fecha, el artículo con mayor número de citas de la revista History of Science.

${ }^{4}$ Las ideas de este apartado están basadas en trabajos previos sobre la genética mendeliana (Casanueva 1987, 2003), y en un artículo escrito en colaboración con Diego Méndez, que enfatiza el uso de imágenes (Casanueva \& Méndez 2008). En la presente formulación, se emplean algunas distinciones propias del programa estructuralista (Balzer, Moulines \& Sneed 1987), sin ahondar en su explicación, cuando se ha considerado necesario, se proporciona una idea intuitiva de las mismas, a medida que aparecen.

Conviene señalar que, tanto en biología, como en la filosofía de la biología, el término "genética mendeliana" se usa de manera ambigua. Para unos es el nombre de una entidad individual (una teoría), para otros refiere a un colectivo que abarca, i.a., los escritos de Mendel, los de sus, llamados, "redescubridores", las versiones divulgadas por Bateson, Punnett o Castle, o las consideraciones precromosómicas sobre el mendelismo de Morgan y su escuela. En aras de la brevedad, esta presentación prescinde de distinciones finas y, es más cercana a lo que en los libros de texto contemporáneos se denomina "mendelismo" o "genética mendeliana" De este estereotipo se han relegado las tesis de la mutación génica, y la consideración de que los genes se agrupan en cromosomas, pues corresponden a desarrollos teóricos distintos. 
Figura 1

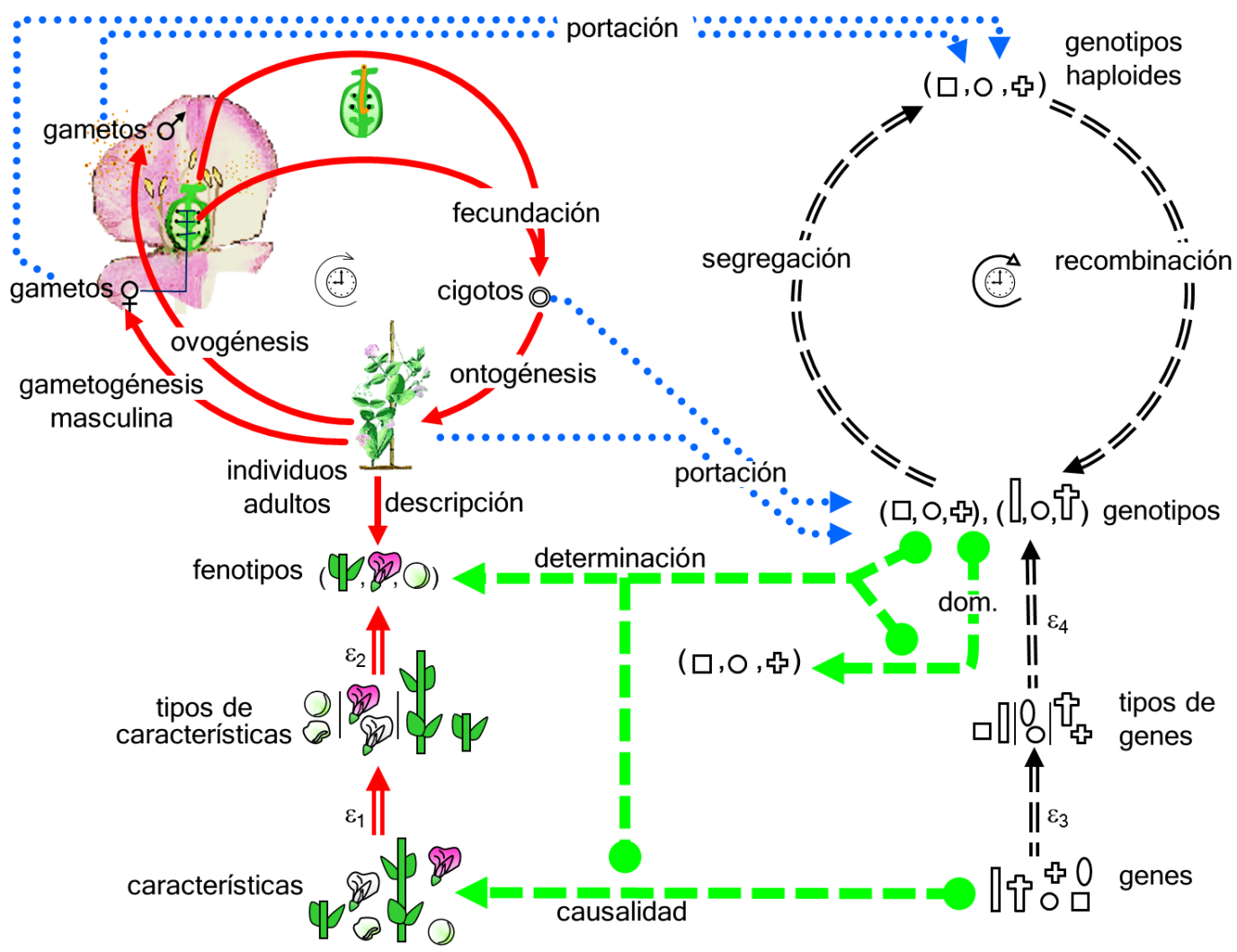

\footnotetext{
$\longrightarrow$ F. rojas continuas sencillas o dobles, permiten la construcción de la pregunta

$\ldots \ldots$ F. azules de puntos, introducen los términos GM-teóricos

$==\Rightarrow$ F. negras de guiones dobles establecen la mecánica del cálculo teórico de la genética mendeliana sobre las frecuencias de genotipos haploides y genotipos diploides

F F verdes de guiones sencillos proporcionan las constricciones que rigen la teoria la flecha dom., sólo está presente en algunos modelos
}

Grafo-modelo de la genética mendeliana (explicación en el texto).

La porción de la izquierda, en la Figura 1, (con las flechas continuas de color rojo, sencillas o dobles) representa, aquellos conceptos que, siendo previos a la teoría, sirven para describir su base de datos o formular la pregunta a la que se trata de dar respuesta. El círculo superior izquierdo con flechas simples, continuas (en rojo), simboliza el ciclo de vida de un organismo de acuerdo con la teoría unigamética de la fecundación (aquí, una planta de chícharo (Pisum sativum), sirve de emblema a los eucariontes sexuados). Los individuos adultos de la generación $t$, producen gametos femeninos ("ovogénesis") y gametos masculinos ("gametogénesis masculina”), las flechas respectivas asocian a cada individuo (singular) el conjunto de gametos masculinos o femeninos por él producidos. La flecha con dos orígenes y un destino marcada "fecundación" representa la unión de los gametos (singamia) y asocia a un par de gametos (masculino y femenino), el cigoto al que su unión da lugar. La flecha rotulada "ontogénesis" representa el desarrollo de un cigoto en un individuo adulto de la generación $\mathrm{t}+1 .^{5}$

\footnotetext{
5 Algunas reconstrucciones estructuralistas (. gr., Balzer \& Dawe 1986, Lorenzano 1995) excluyen toda referencia a los ciclos de vida, y con ello reducen la genética mendeliana a la genética formal; aquí he evitado hacer esto e incluyo explícitamente los referentes a los ciclos de vida, como en su momento, hicieron Mendel o Bateson. Se incluye un ciclo de vida prototípico sin considerar derivaciones del tipo alternancia de generaciones.
} 
A cada individuo se asocia una descripción (fenotipo), en términos de las características heredables que presenta. La parte inferior izquierda muestra los iconos de las distintas características mendelianas a ser consideradas. ${ }^{6}$ La flecha doble, roja, y continua, rotulada $\varepsilon_{1}$, (estructuración 1 ) indica que (a fin de conformar los diferentes fenotipos) las características deben ser agrupadas según su tipo (se representan solo tres tipos, cada uno con dos variantes alélicas). ${ }^{7}$ Un fenotipo es un mosaico de características, una de cada tipo, la flecha denominada $\varepsilon_{2}$ indica este proceso. El interrogante: ¿cómo se transmiten las características a lo largo de las generaciones? puede ser reformulado: ¿qué fenotipo les corresponde a los diferentes descendientes de una determinada pareja (fecundación cruzada), u organismo individual (autofecundación)?

El lado derecho del diagrama, con flechas negras de guiones de línea doble, postula los factores causales de las características (genes alelos) ${ }^{8}$ y establece diferentes formas de ordenamientos y combinatorias de los que participan (lo que podríamos llamar el cálculo teórico). Análogamente a las características, los genes se agrupan según sus diferentes tipos (flecha $\varepsilon_{3}$ ). A continuación, se toma un gen de cada tipo, y se repite esta operación (flecha $\varepsilon_{4}$ ), así, un genotipo diploide consta de un par de mosaicos de genes, cada mosaico tiene un gen de cada tipo. Si para un determinado tipo de gen, ambos mosaicos poseen el mismo alelo, estamos ante un genotipo diploide homocigoto para ese tipo de gen. Si por el contrario, los mosaicos tienen alelos distintos, se trata de un heterocigoto. Un genotipo diploide solo puede presentar dos formas alélicas, aunque en la población puede haber más de dos alelos. Un genotipo haploide posee solo un alelo de cada tipo de gen.

Las flechas denominadas "segregación" y "recombinación" son estructuraciones (líneas dobles) que constituyen la parte central del cálculo de la genética mendeliana. La primera indica, cómo obtener un conjunto de genotipos haploides a partir de un genotipo diploide. La regla es sencilla, un genotipo diploide genera tantos genotipos haploides como posibles sean las combinaciones que se pueden formar tomando un alelo de cada uno de los tipos de genes que lo conforman. La flecha rotulada "recombinación" nos indica cómo obtener genotipos individuales diploides a partir de dos genotipos haploides: basta la simple unión de ambos (en la fecundación, cada gameto aporta un genotipo haploide, y su conjunción (singamia) da lugar al genotipo diploide del cigoto).

Las cuatro flechas azules de líneas de puntos denominadas "portación" permiten introducir los términos genética mendeliana teóricos -esto es, términos propios de la genética mendeliana, cuya correcta aplicación o determinación presupone la validez de las restricciones (flechas verdes) impuestas por el modelo- nos indican el genotipo (haploide o diploide) portado por los gametos, los cigotos y las células somáticas de un individuo adulto, es decir nos indican la descripción genética mendeliana teórica que corresponde a un estado de cosas pre-teórico. Durante la formación de los gametos, los alelos se separan (segregan), por tanto, los gametos (ya sean femeninos o masculinos) portan genotipos haploides. En la fecundación, la unión de los gametos produce nuevamente genotipos diploides cuyos alelos se mantienen juntos hasta la formación de los nuevos gametos en el adulto. Así tanto los cigotos como las células somáticas portan genotipos diploides.

La flecha verde, rotulada "causalidad", vincula genes con características; expresa una idealización que nos indica que característica determinaría cada gen si pudiese expresarse aisladamente. En los casos más sencillos: cada gen alelo se asocia a una y sólo a una característica mendeliana y viceversa, en otros casos como epistasis, pleiotropía o herencia multifactorial, la asociación no es necesariamente uno a uno, y pueden modificarse los números de genes y características involucrados.

La flecha verde, de guiones e inicio redondo rotulada "determinación" vincula genotipos con fenotipos; se trata de una función de funciones, su determinación requiere conocer no sólo el genotipo

\footnotetext{
${ }^{6}$ Operacionalmente, se considera que una característica heredable es mendeliana, si se puede conformar una línea de descendencia cerrada, en la cual la característica permanezca constante a lo largo de las generaciones (cepa pura).

7 No todas las características mendelianas que presenta un individuo son consideradas simultáneamente, aunque podrían serlo. Por lo general, se contemplan de uno a tres pares de características, ocasionalmente incluso decenas, habitualmente la descripción fenotípica es parcial.

8 Frecuentemente, los diferentes tipos de genes poseen más de dos formas alélicas, el término "alelo" deriva de la expresión "gen alelomorfo" (que puede aparecer con diferentes formas). Cada gen alelo es una de las variantes de un tipo de gen, un gen es alelo sólo respecto a otro u otros de su mismo tipo.
} 
en cuestión, sino también el hipotético e idealizado vínculo de causalidad entre genes y características mencionado arriba y algunas funciones adicionales. En el caso más simple, solo se requiere de una función adicional que indica el comportamiento relativo entre los alelos de un mismo tipo de gen (dominante o recesivo). La flecha marcada "dom." ilustra esta función. En los modelos de herencia multifactorial, donde no se contempla una comparación entre alelos, sino un efecto aditivo de sus efectos, en los de pleiotropía, donde u alelo determina más de una característica, y en los de epistasis, donde la presencia de algunos genes puede enmascarar el efecto de otros, que no son sus alelos, se requiere de un mayor número de funciones adicionales, no compartidas con otros tipos de modelos. ${ }^{9}$

\section{El cuadro de Punnett: un diagrama ejemplar}

Desde los Experimentos en híbridos de plantas (Mendel 1866), la distribución de las distintas combinaciones de elementos formativos unidos por la fecundación (hoy "genotipos somáticos diploides"), a partir de los elementos formativos portados por la célula huevo y la célula polen (hoy "genotipos gaméticos haploides") y su expresión como caracteres asociados (hoy fenotipos) se ha presentado mediante algún (algunos) tipo(s) de cuadro(s) o diagrama(s) que permite(n) la construcción de una combinatoria. El desarrollo y evolución de los cuadros de Punnett (introducidos por Bateson, Punnett \& Saunders en 1906) han sido objeto de recientes reflexiones con la intención, i.a., de trazar vínculos históricos con otros diagramas, destacar las ventajas relativas de determinadas formas de cálculo, mostrar su forma de adecuación a diferentes exigencias teóricas y contextos particulares, o relevar su considerable variación temprana y la estabilización convencional de algunos de sus rasgos más frecuentes (p.ej., la posición de relativa de los gametos) (Wimsatt 2012, Edwards 2012,10 2016). Sin embargo, su articulación con el resto de la teoría no ha sido plenamente señalada.

Figura 2

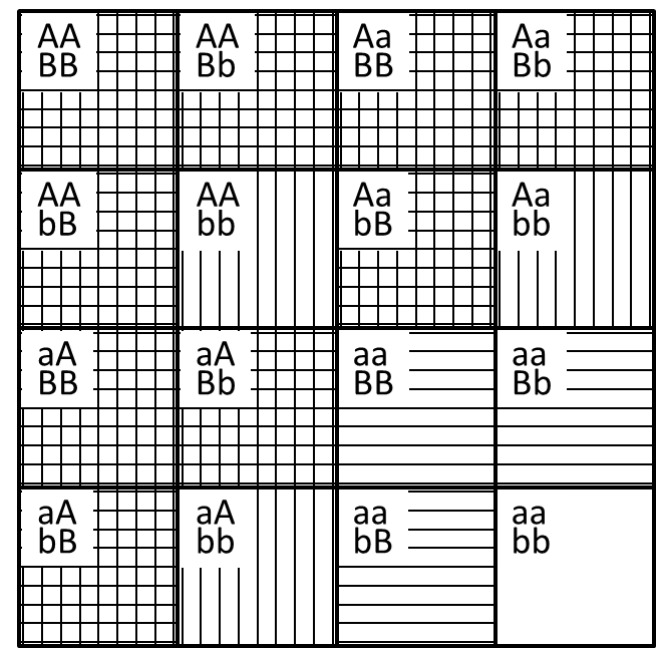

Copia redibujada (con los achurados con mayor distancia entre las líneas) de un temprano cuadro de Punnett (Punnett 1907, p. 45). El diagrama despliega la descendencia genotípica y fenotípica de la cruza de un doble heterocigoto, consigo mismo y muestra la característica proporción fenotípica 9:3:3:1. Cada una de las dieciséis celdas representa un cigoto, las letras simbolizan los diferentes alelos con A dominante sobre $a$ y $B$ sobre $b$, los fenotipos se indican mediante achurados (líneas verticales para el fenotipo $\mathrm{Ab}$, horizontales el $\mathrm{aB}$, cuadrícula para el doble dominante $\mathrm{AB}$, y blanco para el doble recesivo ab). ${ }^{11}$

\footnotetext{
9 Un análisis de las funciones presentes en otros modelos se presenta en Casanueva (2003) y Casanueva \& Méndez (2013).

${ }^{10}$ El texto señala posibles nexos entre el cuadro de Punnett y otros cuadros o diagramas, como los del propio Mendel, o los de Venn, que destacan la presencia (dominancia) o ausencia (recesividad) de determinadas propiedades, entre los miembros de una clase.

${ }^{11}$ Vorms (2014) considera que este tipo de representaciones no refleja ninguna propiedad estructural; en contra, puede señalarse que las celdas contienen a los símbolos que representan los alelos, tal como el cigoto contiene a su genoma o que, de manera más débil, implícitamente, deben ser consideradas como reuniendo alelos contenidos en los dos gametos de los que proceden.
} 
Desde sus orígenes, el cuadro de Punnett ha permitido: "la descomposición visual natural de un problema complejo en una serie de etapas relacionadas entre si”" (Wimsatt 2012, p. 359), lo que permite el tratamiento sistemático de diversos tipos de problemas y da cuenta de su potencia conceptual y de cómputo.

El diagrama permite al menos dos diferentes estrategias cognitivas de llenado (una celda puede ser llenada por varias vías de acceso que son lógicamente equivalentes, pero cognitivamente distintas), la primera sigue una lógica matricial de cruces de información gamética invariante (renglón por columna o viceversa), la segunda está basada en una lógica de subdivisión jerárquica de áreas (recursiva-fractal) (Wimsatt 2012). Un cuadrado inicial se divide tanto horizontal como verticalmente en dos zonas (digamos A y a), ello da lugar a cuatro cuadrados que corresponden a las combinaciones para el primer par de alelos (AA, Aa, aA, y aa), cada uno se divide a su vez en otros cuatro (que corresponden a las combinaciones para el segundo par de alelos (digamos, BB, Bb, bB y bb), de esta forma, por ejemplo, el cuadrado AA queda dividido en cuatro zonas: AABB, AABb, AAbB y AAbb y lo mismo ocurre con los primeros cuatro cuadrados, resultando dieciséis cuadrados que se pueden volver a dividir en las cuatro combinaciones a que da lugar el tercer par de alelos... y así recursivamente.

Inicialmente los cuadros de Punnett eran similares a la figura dos, mostraban sólo el resultado de la combinatoria genética y los fenotipos esperados, según la especialización concreta representada (v.g., dominancia estricta, codominancia o alguna de las múltiples formas de la epistasis). Actualmente es frecuente encontrar en ellos, una cantidad significativa de trazos subsidiarios que añaden o explicitan información, (por ejemplo, el significado genético de renglones y columnas). ${ }^{12}$ Véase Figura 3.

El lado izquierdo de la Figura 3 muestra un cuadro de Punnett contemporáneo, a la matriz combinatoria, ejemplificada por la Figura 2, se ha añadido información subsidiaria y se han cambiado algunas convenciones. La matriz se representa inclinada, los alelos están representados por símbolos y no por letras, los fenotipos se muestran mediante íconos que, a diferencia de los distintos achurados, guardan semejanza con la característica que representan, pero las celdas siguen representando cigotos. Respecto a las adiciones: el recuadro arriba a la izquierda, indica tanto la apariencia fenotípica como la constitución genotípica de un organismo doblemente heterocigoto que se cruzará consigo mismo o con otro semejante (a veces, se incluye información acerca de su procedencia, normalmente, se trata de un dihíbrido resultado de la cruza de un doble homocigoto dominante con un doble homocigoto recesivo). Las flechas de puntos azules muestran la procedencia de las entradas de la matriz (los genotipos haploides portados por los gametos femeninos del lado izquierdo, y masculinos, del derecho). Las entradas mismas, antes ausentes, constituyen los genotipos haploides portados por los gametos femeninos o masculinos (al igual que las celdas, preservan la relación estructural contenido continente que se da entre los genes y los gametos o cigotos). Se ha agregado información sobre las frecuencias relativas de los diferentes tipos de gametos y de los genotipos obtenidos por su unión y finalmente el recuadro inferior compendia la información sobre las proporciones fenotípicas.

El lado derecho de la imagen descompone las operaciones necesarias para la obtención de la matriz y señala su correspondencia con diferentes funciones en la estructura lógico conceptual de la genética mendeliana (Figura 1). Se ha respetado la clave de los distintos tipos de flechas señalada en la Figura 1. La recombinación se ha desdoblado en dos matrices, la primera indica el cálculo frecuencialista, la segunda muestra la ocurrencia de los diferentes alelos en gametos y cigotos. Una tercera matriz, indica los fenotipos correspondientes a los genotipos de la segunda matriz (flecha marcada determinación). Las tres matrices se encuentran vinculadas en el cuadro de Punnett. Dado que las celdas representan cigotos su perímetro se ha representado por líneas dobles, rojas.

\footnotetext{
${ }^{12}$ El diagrama de Punnett permite la incorporación de información cromosómica o incluso han sido usados como herramientas de análisis metapoblacional (Griesemer \& Wade 2000).
} 
Figura 3

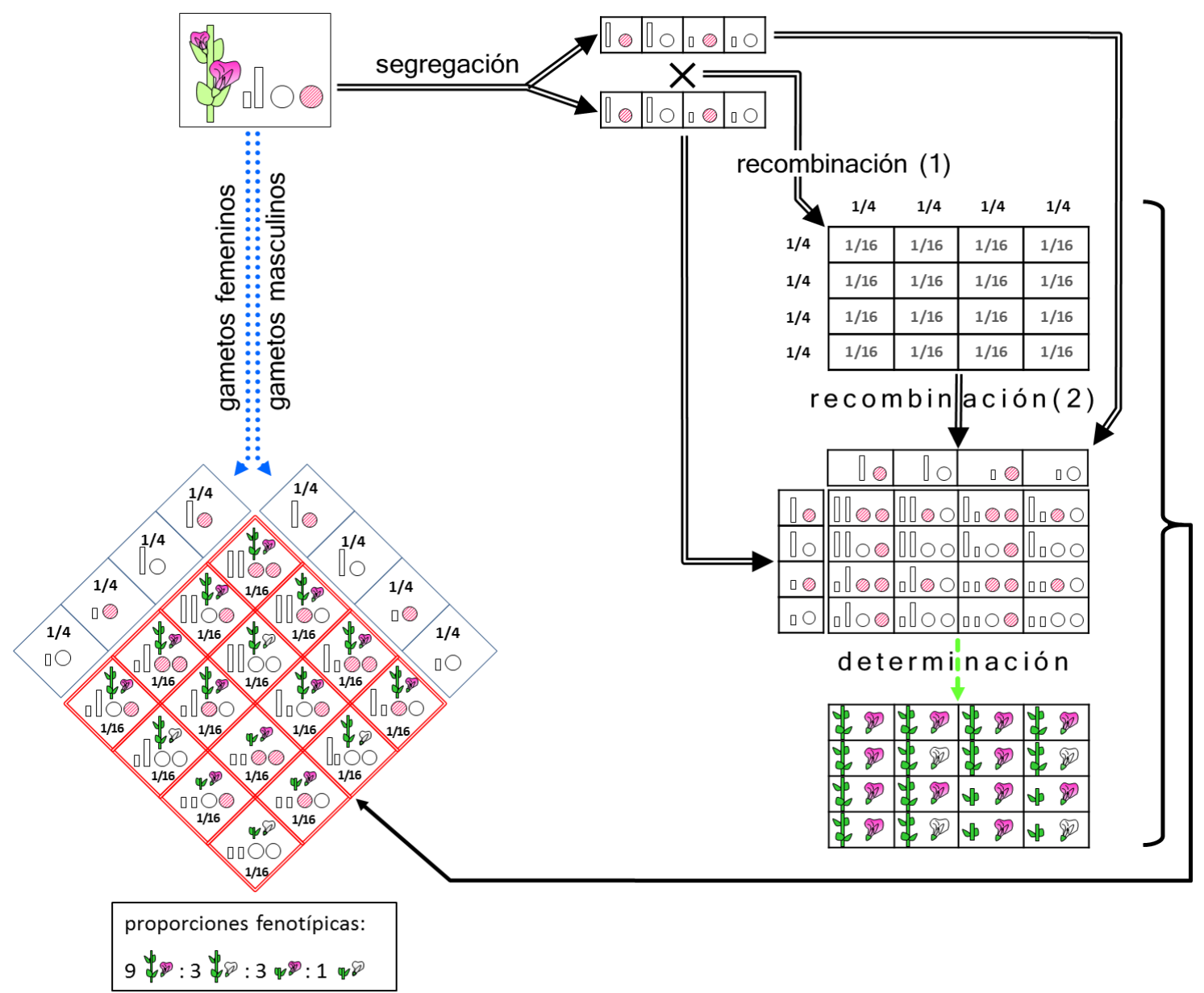

Versión contemporánea de un cuadro de Punnett (lado izquierdo) y descomposición del mismo en distintos momentos y funciones involucrados en su construcción (lado derecho). Explicación en el texto.

\section{Conclusiones}

La comparación de la estructura subyacente a los cuadros de Punnett y la arquitectura conceptual de la genética mendeliana muestran con claridad la manera en que los cuadros de Punnett se encuentran articulados a la estructura de la genética mendeliana. En sus versiones actuales presentes en libros de texto y numerosas páginas web los cuadros de Punnett incluyen elementos de los cuatro momentos epistémicos presentes en la teoría: (i) La formulación de la pregunta implícita sería: ¿cuál es la naturaleza de los cigotos que proceden de la cruza de organismos con genotipos conocidos? (ii) Los términos genética mendeliana-teóricos se introducen mediante el señalamiento de cuáles son los genes que portan los gametos. (iii) La parte del cálculo teórico constituye el aspecto más destacado del diagrama pues recupera tanto la idea de la segregación como la recombinación independiente. Y (iv) las leyes de la determinación genética se compendian al traducir combinaciones genéticas a combinaciones de caracteres dentro de cada celda. Así, puede señalarse que, esencialmente, un cuadro de Punnett es un modelo de una subestructura de la genética clásica que representa los aspectos más destacados de su propuesta teórica. Constituye, por así decirlo un mini-resumen de la estructura de la teoría que sistematiza, y facilita mediante la construcción de un proceso estandarizado y multirrealizable, la obtención de las diferentes frecuencias genotípicas y fenotípicas a que da lugar la cruza de dos genotipos dados, al tiempo que permite la adición de información con contenido estructural. Son estas características las que le confieren su gran capacidad como herramienta para el desarrollo y enseñanza de la genética clásica. 
Abrahamsen, A. y W. Bechtel (2015), “Diagrams as Tools for Scientific Reasoning”, Review of Philosophy and Psychology 6(1): $117-131$.

Aglioti, S.M., Bufalari, I. y M. Candidi (2014), "Multisensory Mental Simulation and Aesthetic Perception”, en Levent, N. y A. Pascual-Leone (eds.), The Multisensory Museum: Cross-Disciplinary Perspectives on Touch, Sound, Smell, Memory, and Space, Lanham: Rowman \& Littlefield, pp. 301-318.

Ainsworth, S., Prain, V. y R. Tytler (2011), “Drawing to Learn in Science”, Science 333(6046): 1096-1097.

Auden, W.H. y E. Mayer (1962), Goethe, J. W. Italian Journey (1786-1788), London: Penguin Classics.

Balzer, W. y C.M. Dawe (1986), "Structure and Comparison of Genetic Theories: (I) Classical genetics", The British Journal for the Philosophy of Science 37(1): 55-69.

Balzer, W., Moulines, C.U. y J.D. Sneed (1987), An Architectonic for Science. The Structuralist Program, Dordrecht: Reidel.

Bateson, W., Punnett. R.C. y E. Saunders (1906), Experimental Studies in the Physiology of Heredity (Report to the Evolution Committee of the Royal Society, III), London: The Royal Society.

Bechtel, W. y A. Abrahamsen (2005), "Explanation: A Mechanist Alternative", Studies in History and Philosophy of Biological and Biomedical Sciences 36: 421-441.

Bechtel, W. y R.C. Richardson (1993), Discovering Complexity: Decomposition and Localization as Strategies in Scientific Research, Princeton: Princeton University Press.

Binder, E., Hagelweide, K., Wang, L.E., Kornysheva, K., Grefkes, C., Fink, G.R. y R.I. Schubotz (2014), "SensoryGuided Motor Tasks Benefit from Mental Training Based on Serial Prediction”, Neuropsychologia 54: 18-27.

Burnston, D.C. (2016), "Data Graphs and Mechanistic Explanation”, Studies in History and Philosophy of Science Part C: Studies in History and Philosophy of Biological and Biomedical Sciences 57: 1-12.

Burnston, D.C., Sheredos, B., Abrahamsen, A. y W. Bechtel (2014), "Scientists' Use of Diagrams in Developing Mechanistic Explanations: A Case Study from Chronobiology”, Pragmatics $\mathcal{E}$ Cognition 22(2): 224-243.

Casanueva, M. (1987), "La estructura lógica de la genética clásica”, Memorias del LVII Congreso de la Asociación de Investigación Pediátrica (México), Vol. I: 99-108.

Casanueva, M. (2003), Mendeliana, Iztapalapa, México: Editorial M.A. Porrúa-Biblioteca de Signos, UAM.

Casanueva, M. (2005), "Los modelos en la filosofía de la ciencia del siglo XX”, en López Austín A. (ed.), El modelo en la ciencia y la cultura, México: Siglo XXI, pp. 29-53.

Casanueva, M. y D. Méndez (2008), “Teoría y experimento en genética mendeliana: una exposición en imágenes”, Theoria 63: 285-306.

Casanueva, M. y D. Méndez (2013), "Graphs and Lattices as Conceptual Representation Formats. An Exploratory Exercise with Five Specializations of Mendelian Genetics", Metatheoria 3(1): 19-37.

Chandrasekharan, S. y N.J. Nersessian (2015), "Building Cognition: The Construction of Computational Representations for Scientific Discovery”, Cognitive science 39(8): 1727-1763.

Cheon, H. (2014), "Distributed Cognition in Scientific Contexts”, Journal for General Philosophy of Science 45(1): 23-33.

Craver, C.F. y M.I. Kaiser (2013), "Mechanisms and Laws: Clarifying the Debate”, en Chao, H., Chen, S. y R. Millstein (eds.), Mechanism and Causality in Biology and Economics, Dordrecht: Springer, pp. 125-45.

Darden, L. y C. Craver (2002), "Strategies in the Interfield Discovery of the Mechanism of Protein Synthesis", Studies in History and Philosophy of Science Part C: Studies in History and Philosophy of Biological and Biomedical Sciences 33(1): 1-28.

Daston, L. y P. Galison (1992), "The Image of Objectivity”, Representations 40: 81-128.

Díez, J.A. y C.U. Moulines (1999), Fundamentos de la filosofía de la ciencia, Barcelona: Ariel.

Edwards, A.W.F. (2012), "Punnett's Square", Studies in History and Philosophy of Science Part C: Studies in History and Philosophy of Biological and Biomedical Sciences 43(1): 219-224.

Edwards, A.W.F. (2016), "Punnett's Square: A Postscript”, Studies in History and Philosophy of Science Part C: Studies in History and Philosophy of Biological and Biomedical Sciences 57: 69-70. 
Ferri, F., Frassinetti, F., Ardizzi, M., Costantini, M. y V. Gallese (2012), “A Sensorimotor Network for the Bodily Self”, Journal of Cognitive Neuroscience 24(7): 1584-1595.

Galison, P. (2006), "Images Scatter into Data, Data Gather into Images”, en Manghani, S., Piper, A. y J. Simons (eds.), Images: A Reader, London: Sage, pp. 236-41.

Gebharter, A. y M.I. Kaiser (2013), “Causal Graphs and Biological Mechanisms”, en Kaiser, M.I., Scholz, O.R., Plenge, D. y A. Hüttemann (eds.), Explanation in the Special Sciences, Dordrecht: Springer, pp. 55-85.

Giere, R.N. (1988), Explaining Science: A Cognitive Approach, Chicago: University of Chicago Press.

Giere, R.N. (2004), "How Models are Used to Represent Reality”, Philosophy of Science 71(Supplement): $742-752$.

Giere, R.N. (2010), “An Agent-Based Conception of Models and Scientific Representation”, Synthese 172(2): $269-281$.

Glennan S. (2002), “Rethinking Mechanistic Explanation”, Philosophy of Science 69: 342-53.

Griesemer, J.R. y M.J. Wade (2000), "Populational Heritability: Extending Punnett Square Concepts to Evolution at the Metapopulation Level”, Biology and Philosophy 15(1): 1-17.

Griesemer, J.R. y W.C. Wimsatt (1989), "Picturing Weismannism: A Case Study in Conceptual Evolution”, en Ruse, M. (ed.), What Philosophy of Biology Is (Essays Dedicated to David Hull), Dordrecht-Boston-London: Kluwer, pp. $75-$ 137.

Jones, N. (2014), “Bowtie Structures, Pathway Diagrams, and Topological Explanation”, Erkenntnis 79(5): 1135-1155.

Jones, N. y O. Wolkenhauer (2012), "Diagrams as Locality aids for Explanation and Model Construction in Cell Biology”, Biology Ë Philosophy 27(5): 705-721.

Kozma, R. (2003), "The Material Features of Multiple Representations and their Cognitive and Social Affordances for Science Understanding", Learning and Instruction 13(2): 205-226.

Lakoff, G. y M. Johnson (1980), Methaphors We Live By, Chicago: University of Chicago Press.

Lakoff, G. y M. Johnson (1999), Philosophy in the Flesh: The Embodied Mind and its Challenge to Western Thought, New York: Basic Books.

Larkin, J.H. y H.A. Simon (1987), "Why a Diagram is (Sometimes) Worth Ten Thousand Words”, Cognitive Science 11(1): 65-100.

Lorenzano, P. (1995), Geschichte und Struktur der klassischen Genetik, Frankfurt am Main: Peter Lang.

Mast, F.W. y A.W. Ellis (2015), "Internal Models, Vestibular Cognition, and Mental Imagery: Conceptual Considerations”, Multisensory Res 28(5-6): 443-460.

Mendel, J.G. (1866), "Versuche über Pflanzen-hybriden”, Verhandlungen des Naturforschenden Vereins zu Brünn, Abhandlungen, iv. 1865. (Traducción inglesa en: Stern, C. y E.R. Sherwood (eds.), The Origin of Genetics, San Francisco: W. H. Freeman, 1966, pp. 3-49.)

Méndez, D. y M. Casanueva (2006), “A Reconstruction of Darwin's Pangenesis in a Graph Format”, en Ernst, G. y K.-G. Niebergall (eds.), Philosophie der Wissenchaft - Wissenschaft der Philosophie, Paderborn: Mentis, pp. 157-164.

Moreau, D. (2012), "The Role of Motor Processes in Three-Dimensional Mental Rotation: Shaping Cognitive Processing Via Sensorimotor Experience”, Learning and Individual Differences 22(3): 354-359.

Osbeck, L.M. y N.J. Nersessian (2014), “Situating Distributed Cognition”, Philosophical Psychology 27(1): 82-97.

Perini, L. (2005), “Explanation in Two Dimensions: Diagrams and Biological Explanation”, Biology and Philosophy 20(23): $257-269$.

Perini, L. (2013), "Diagrams in Biology”, The Knowledge Engineering Review 28(3): 273-286.

Punnett, R.C. (1907), Mendelism, $2^{\text {nd }}$ edition, Cambridge: Bowes and Bowes.

Rudwick, M.J. (1976), “The Emergence of a Visual Language for Geological Science 1760-1840”, History of Science 14(3): 149-195.

Sheredos, B., Burnston, D., Abrahamsen, A. y W. Bechtel (2013), "Why Do Biologists Use So Many Diagrams?", Philosophy of Science 80(5): 931-944. 
Sollfrank, T., Hart, D., Goodsell, R., Foster, J. y T. Tan (2015), “3D Visualization of Movements Can Amplify Motor Cortex Activation During Subsequent Motor Imagery”, Frontiers in human neuroscience 9(463): 1-8.

Suppe, F. (1977), "The Search for Philosophic Understanding of Scientific Theories", en Suppe, F. (ed.) The Structure of Scientific Theories, Urbana: University of Illinois Press, pp. 1-244.

Tversky, B. (2011), "Visualizing Thought", Topics in Cognitive Science 3(3): 499-535.

Tversky, B. y M. Suwa (2009), “Thinking with Sketches”, en Markman, A.B. y K.L. Wood (eds.), Tools for Innovation: The Science Behind the Practical Methods that Drive New Ideas, New York: Oxford University Press, pp. 75-84.

Tversky, B., Heiser, J., Lee, P. y M.P. Daniel (2009), "Explanations in Gesture, Diagram, and Word”, en Coventry, K.R., Tenbrink, T. y J. Bateman (eds.), Spatial Language and Dialogue, Vol. 3, Oxford: Oxford University Press, pp. $119-131$.

Tversky, B., Zacks, J., Lee, P. y J. Heiser (2000), "Lines, Blobs, Crosses and Arrows: Diagrammatic Communication with Schematic Figures", en International Conference on Theory and Application of Diagrams, Berlin-Heidelberg: Springer, pp. 221-230.

Tylén, K., Fusaroli, R., Bjørndahl, J.S., Raczaszek-Leonardi, J. y F. Stjernfelt (2014), "Diagrammatic Reasoning: Abstraction, Interaction, and Insight", Pragmatics $\mathcal{E}$ Cognition 22(2): 264-283.

Van Fraassen, B.C. (1970), “On the Extension of Beth’s Semantics of Physical Theories”, Philosophy of Science 37: 667698.

Vorms, M. (2013), “Theorizing and Representational Practices in Classical Genetics", Biological Theory 7(4): 311-324.

Vorms, M. (2014), "The Birth of Classical Genetics as the Junction of Two Disciplines: Conceptual Change as Representational Change", Studies in History and Philosophy of Science Part A 48: 105-116.

Wimsatt, W.C. (2012), "The Analytic Geometry of Genetics: Part I: the Structure, Function, and Early Evolution of Punnett Squares”, Archive for History of Exact Science 66(4): 359-396.

Zenker, F. y P. Gärdenfors (2015), “Communication, Rationality, and Conceptual Changes in Scientific Theories”, en Zenker, F. y P. Gärdenfors (eds.), Applications of Conceptual Spaces, Heidelberg-New York-Dordrecht-London: Springer, pp 259-277. 